\title{
The Long-term Light Curves of X-ray Binaries Contain Simultaneous Periodic and Random Components
}

\author{
Patricia T. Boyd ${ }^{1}$ \\ Alan P. Smale ${ }^{2}$ \\ Laboratory for High Energy Astrophysics, Code 662, NASA/Goddard Space Flight Center, Greenbelt, MD 20771
}

\begin{abstract}
LMC X-3 and Cyg X-2 show large amplitude X-ray fluctuations that have been attributed to a warped accretion disk. Cyg X-3 displays high amplitude, apparently nonperiodic oscillations. We reanalyze these systems using RXTE ASM data and timefrequency decomposition techniques. We find that the long-term variations in Cyg X-2 can be completely characterized by excursions whose durations are integer multiples of the orbital period, including one essentially identical to the reported "period" of 78 days. Cyg X-3 can be characterized in terms of integer multiples of a 71-day fundamental period, unrelated to the 4.8 day orbital period, but suggestively close to the $\gtrsim 60$ day reported precession period of the relativistic jet inferred from recent radio observations. The long-term excursions of LMC X-3 are related to each other by rational fractions, suggesting the characteristic time scale is 10.594 days, shorter than any observed excursion to date. We explore the phase space evolution of the light curves using a natural embedding and find that all three systems possess two rotation centers that organize the phase space trajectories, one of low luminosity and the other of high luminosity. The implications of this repeatable behavior on generic models of accretion disk dynamics and mass transfer variability are explored.
\end{abstract}

Subject headings: accretion, accretion disks - stars: individual (LMC X-3, Cyg X -2 , Cyg X-3) - stars: binaries: close - X-rays: stars

$$
\begin{aligned}
& \text { Submitted to the Astrophysical Journal } \\
& \text { Sept. } 30,2002
\end{aligned}
$$

\footnotetext{
'Also Joint Center for Astrophysics, University of Maryland Baltimore County

${ }^{2}$ Also Universities Space Research Association
} 


\section{Introduction}

Several binary star systems with X-ray emitting companions show large amplitude variations in their $\mathrm{X}$-ray light curves on timescales significantly longer than their orbital periods. Her X-1, an accreting pulsar, displays a double-peaked 35 -day X-ray variation with properties that are successfully explained by the presence of a warped or inclined accretion disk that precesses with respect to the binary orbital plane (Priedhorsky \& Holt 1987). Other systems showing nearly periodic variations an order of magnitude longer than the orbital period include the accreting X-ray pulsars SMC X-1 (50-60 day period; Gruber \& Rothschild 1984, Wojdowski et al. 1998), and LMC $\mathrm{X}-4$ (30.5 day period, Lang et al. 1981 ), as well as the paradigmatic disk-jet source SS 433 (Fabian \& Rees 1979, Abell \& Margon 1979). The similarities in their long-term evolutions, coupled with the elegant precessing jet model to explain the observed properties of SS 433 , led researchers to consider these objects together as a class, in which precessing accretion disks give rise to "superorbital periodicities". Other systems were found to have candidate long term periods (LMC X-3; Cowley et al. 1991, and Cyg X-2; Smale \& Lochner 1992), based on sparse, unevenly sampled data, and were added to the class. (While a simple precessing disk model predicts strict periodicity, even one of the most convincing sources, Her $\mathrm{X}-1$, has occasionally entered prolonged "anomalous low states" in which the long-term X-ray variation is not detected.)

We have examined the light curves of several X-ray binaries which show long-term variability using data from the All-Sky Monitor (ASM) aboard the Rossi $\mathrm{X}$-ray Timing Explorer. Here, we concentrate on three systems with very different basic properties-the black hole candidate LMC X-3, the low mass Xray binary neutron star Z-source Cyg $\mathrm{X}-2$, and the enigmatic radio jet source Cyg $\mathrm{X}-3$. We find that none shows strict periodicity for even two consecutive putative cycles. By characterizing the light curves as a series of excursions from one X-ray low state to the next, we find that the excursion times can be related to one another via low order, but apparently random, integer multiples.

The organization of this paper is as follows. In section 2, we review the known geometric characteristics of each system, and our time series analysis methods. Section 3 contains the results of this analysis for each source. In section 4 , we compare the phase space trajectories of the X-ray binary systems with the driven Duffing oscillator and magnetic disk models. We also discuss the implications of this behavior, draw parallels to more familiar physical systems, and suggest a possible model for the long-term variability of the sources due to accretion and geometry, incorporating both random and predictable components. Our conclusions on the existence of simultaneous random \& predictable components in the long-term light curves of X-ray binaries are presented in section 5 .

\section{Systems \& Analysis}

The bright, persistent, low mass X-ray binary Cyg $\mathrm{X}-2$ is composed of a neutron star (Orosz \& Kuulkers 1999) in a 9.844 day orbit (Cowley, Crampton, \& Hutchings 1979; Casares, Charles, \& Kuulkers 1998) with its companion V1341 Cyg. It traces out a Zshape on a color-color diagram on timescales of about one day. It displays type I X-ray bursts (Kahn \& Grindlay 1984; Smale 1998) and intensity dips (Vrtilek et al. 1988), as well as quasiperiodic oscillations from $\mathrm{Hz}$ to $\mathrm{kHz}$ frequencies. A 77 -day periodicity was reported in Vela 5B data (Smale \& Lochner 1992). Wijnands, Kuulkers \& Smale (1996) found a 78 day period from analysis of the first 160 days of RXTE/ASM data, combined with archival data from Vela 5B and Ariel 5. Later, Paul, Kitamoto \& Makino (2000) found that the 78 day period does not endure through longer stretches of the ASM light curve.

LMC X-3 is a bright (up to $3 \times 10^{38} \mathrm{erg} \mathrm{s}^{-1}$ ) highly variable $\mathrm{X}$-ray source in the Large Magellanic Cloud. It exhibits two distinct emission states: the more common high/soft state, in which the X-ray spectrum has an "ultrasoft" component and a hard (>10keV) tail (similar to other high/soft state black hole candidates) ; and the low/hard state, characterized by an $\mathrm{X}$-ray spectrum described by a pure power law $I(E)=$ $A_{p l} E^{-\Gamma}$ with $\Gamma \sim 1.8$ or less (Wilms et al. 2001), significant time variability with $30-50 \%$ modulation of the intensity, and a $0.4 \mathrm{~Hz}$ QPO (Boyd et al. 2000). The B3 V optical counterpart (Warren and Penfold 1975) ( $V \sim 16.7-17.5$ ) shows a large velocity range (semiamplitude $\mathrm{K}=235 \mathrm{~km} \mathrm{~s}^{-1}$ ) through its 1.7 day orbital period. The lack of eclipses indicates that the inclination of the system is $<70^{\circ}$, leading to a compact object mass of $\sim 7 \mathrm{M}_{\odot}$, making LMC X -3 a prime black hole candidate (Cowley et al. 1983, Paczynski 1983, Ebisawa et al. 1993, but see also Mazeh et al. 
1986): The long-term X-ray luminosity of the system is strongly modulated on time scales of hundreds of days (Cowley et al. 1991, Wilms et al. 2001). The mean $2-10 \mathrm{keV}$ X-ray flux varies by a factor of more than one hundred during this long-term cycle (Boyd et al. 2000). Although this variability was previously attributed to the precession of a bright, tilted, and warped accretion disk, the discovery of recurrent low/hard states in LMC X-3 suggests additional mechanisms may be responsible for the longterm variation (Wilms et al. 2001).

Cyg $\mathrm{X}-3$ is an unusual object in many respects. It is one of only a few X-ray binaries that is a persistently strong emitter of radio waves, and is perhaps the strongest radio emitter of all X-ray binaries (Waltman et al. 1995). The companion is very likely a Wolf-Rayet star with a strong, optically thick stellar wind. The compact object orbits this star every 4.8 hours, virtually within the companion's outer layers. While it is not conclusively known whether the compact object is a BH (see Schmutz, Geballe \& Schild 1996 who estimate compact object mass at $>7$ solar masses) or NS (Hanson, Still \& Fender 2000, Mitra 1998) the existence of giant radio flares and strong, relativistic and variable radio jets is reminiscent of the (X-ray transient) galactic microquasar sources GRS 1915+105 and GRO J1655-40. Mioduszewski et al. (2001) fit a precessing jet model to VLBA data of a curved radio jet taken after a flare and find best fit model parameters suggesting a precession period of $Z 60$ days. Aside from this, we are aware of no other claims of candidate long-term periods in Cyg X-3.

The All Sky Monitor (ASM; Levine et al. 1996) aboard the Rossi X-ray Timing Explorer (RXTE; Bradt, Rothschild \& Swank 1993) is a set of three scanning shadow cameras covering the $1.5-12.0 \mathrm{keV}$ energy range in three energy bands. It scans most of the sky each 90-minute satellite orbit, collecting $\mathrm{X}$-ray intensity measurements in 90-second dwells. Launched in December 1995, with a large continuous viewing zone, the ASM has generated an impressive archive of high quality, evenly sampled time series data on virtually all moderately bright X-ray sources with a nearly seven-year baseline. The ASM light curves can be used to explore variability timescales from days to years.

For each of our three sources of interest, we generated ASM light curves with a sample time of 1-day. Data points with error bars greater than three times the average error were eliminated. These points, as well as short, sporadic gaps in the data, were replaced with linearly interpolated values, resulting in evenly spaced time series data. In all cases, the randomly spaced inserted data comprise $15 \%$ or less of the total data points. The ASM light curves begin at JD2450087 and end on JD2452031.

The standard Fast Fourier Transform (FFT) was used on the resulting evenly space time series to generate power spectra. A numerically generated evenly spaced time derivative was used to explore the behavior of each system in a natural phase space.

\section{Results}

In this section we present the results or our time domain analysis of the long-term variations in Cyg X2 , LMC X-3 and Cyg X-3. No stable long-term period is found in any of the three systems. We characterize each time series in terms of excursions between minima, and find the excursion times are related by integers.

\subsection{Cyg $\mathrm{X}-2$}

Cyg X-2 has been the object of several long-term variability studies based on data from the RXTE ASM and other monitoring X-ray missions such as Ginga, ARIEL 5, and Vela 5B. Smale \& Lochner (1992) reported the detection of a significant periodicity of 77.34 days in Vela 5B data. This was seen again in the first 160 days of RXTE ASM monitoring of the source (Wijnands, Kuulkers \& Smale, 1996). Later, Kong, Charles \& Kuulkers (1998) reported a more significant detection of a 69 day periodicity, based on 800 days of RXTE ASM data. Paul, Kitamoto \& Makino (2000) analyzed 1000 days of RXTE ASM data in which they did not detect a 77 day periodicity with statistical significance. Instead, they report detections at 68.8 days and 40.4 days in the data set as a whole. By separating the time series into three approximately 1-year stretches and generating separate periodograms, they showed that the frequencies and amplitudes vary with time.

The ASM daily average count rate for Cyg $\mathrm{X}-2$ is plotted versus time in Figure 1a. The quality of the ASM light curve for Cyg X-2 is quite high. The oneday average count rate is about 38 counts $\mathrm{s}^{-1}$. There are typically 5-10 individual ASM dwells per day on the source. Errors on the one-day average points are the quadrature average of the errors on the individual dwells. The typical value for the one-day error is 0.55 
counts $\mathrm{s}^{-1}$.

The apparent contradictions of the three aforementioned variability studies of Cyg $\mathrm{X}-2$, all based on periodogram analysis of the entire data set, strongly implies that the long-term light curve of $\mathrm{Cyg} \mathrm{X}-2$ contains time-evolving (as opposed to fixed) frequencies. If such is the case, more can be learned from a time domain analysis than from a Fourier analysis, generally appropriate for extracting fixed frequencies in time series data.

We first attempted to confirm and unify the results of the previous analyses, first by using direct FFTs on 5.5 years of very nearly evenly spaced ASM data. We generated a PSD of the 1-day average ASM light curve by creating an evenly sampled time series from all available data with error bars less than three times the average error (Figure 1). Where no data were present (about $11 \%$ of the total time series, in randomly spaced, few-day segments) we linearly interpolated between data points. The resulting evenly spaced time series contains 1944 total data points, of which 226 are interpolated points. We padded this series with random data with the same mean and standard deviation as the original data set, out to a length of 2000 points.

Next, we divided this time series into two equal 1000-day segments, subtracted the mean value, and generated the power spectrum of each with a standard FFT routine. At each of the 500 independent frequencies, we took the average value of the two power spectra. This method involves no interpolation or smoothing between adjacent frequencies and thus preserves only the frequency information available in the data at the 1-day sampling resolution. We normalized the individual powers by dividing them by the total power in all frequencies. With this normalization, we can directly ascertain the significance of multiple spectral peaks via the Fisher criterion (Fisher 1929, Shimshoni, 1971). The resulting power spectrum is shown in Figure 1b.

For a random data set of 1000 points, the probability that its power spectrum will contain a single peak as high or higher than 0.026 is $P=0.001$. At least six peaks lie above this significance level in the average PSD displayed in Figure 1b. One peak has a corresponding period of 78.8 days (the Wijnands period), but it is not the highest peak in the power spectrum. The highest peak, with a period of 68.9 days (the Kong period, and one of the Paul periods), contains significantly more power in the PSD. These, and other significant frequencies in the PSD, are marked with vertical lines in Figure Ib.

There are several immediately intriguing features in the PSD shown in Figure 1b. First is that the 78 day period reported by Smale \& Lochner (1992) in Vela $5 \mathrm{~B}$, and later by Wijnands, Kuulkers \& Smale (1996) in the RXTE ASM and other data sets is present, but is not the most significant peak. It is surprising that this single periodicity was present in (admittedly sparse) data spanning several decades, yet does not endure as the single periodicity in the ASM, a high S/N, long time-baseline data set.

Even more intriguing is the fact that the most significant period, 68.9 days, differs from the 78.7 day period reported in the earlier data sets by, within the frequency resolution, exactly 1 binary orbit $(9.844$ days). The 68.9 day period is seven binary orbits, while the 78.7 day periodicity is 8 binary orbits. In fact, all of the significant peaks in Figure $1 \mathrm{~b}$ are simply integer multiples of the binary period, within the frequency resolution of the PSD. (Note that no significant feature at the binary period is found.) To the left of each vertical line in the PSD of Figure $1 \mathrm{~b}$, the integer number of binary cycles corresponding to that frequency is indicated. Table 1 contains the lengths of these integers multiplied by the binary period, and their corresponding absolute frequencies.

What kind of time series gives rise to multiple peaks in the power spectrum, at integer multiples of the binary orbit - a reliable dynamical clock in the system? To answer this question, we focused again on the time domain and compared the long-term light curve to a sinusoidal function representing the binary phase, two representative segments of which are shown in Figure 2. We arbitrarily chose a fiducial phase to line up with the first turning point in the long-term light curve.

The Cyg X-2 ASM light curve has some turning points that occur at lower count rates than others. We refer to these as "deep" minima if they drop lower than 30 ASM counts $\mathrm{s}^{-1}$. We find that most deep minima in the long-term light curve of $\mathrm{Cyg} X-2$ line up with the minima in the binary phase function as shown in Figure 2. Using the binary orbit as an integer counter, we were next able to characterize the entire long-term light curve of $\mathrm{Cyg} \mathrm{X}-2$ as a series of excursions whose lengths $P_{i}$ are integer multiples $N_{1}$ of the binary period $P_{0}=9.844$ days. From the 36 excursions studied, it does not appear possible to predict the next value of $N_{i}$ from the knowledge of 
the previous values $N_{0}$ through $N_{i-1}$. Instead, $N_{i}$ varies in an apparently random way. The long-term lightcurve of Cyg X-2 must be caused by a physical process or processes that give rise to the multiplication of a periodic component (the binary period) by a random integer. If the times of deep minima are aligned closely with a repeating geometric orientation of the binary, it is plausible that the deep minima are somehow tied to the orbital geometry of the system. Another process at work in Cyg $\mathrm{X}-2$ must give rise to the random component.

Different random processes give rise to different statistical distributions. Measureable properties of a specific distribution can give meaningful insight into the process which is generating the random distribution. The distribution of excursion times $N_{i}$, is shown in Figure 3a, along with a Gaussian fit. The actual distribution more closely resembles a Poisson, as expected from a random distribution. As more ASM data is collected, and more trials are available from which to build a reliable statistical distribution, the true form of the distribution function will emerge.

There is a slight drift in the binary phase at which the deep minima occur. This drift does not appear to be randomly distributed about phase zero, but evolves smoothly. We show this in Figure $3 \mathrm{~b}$, where we have plotted the binary phase at which the actual deep minimum occurs versus $i$, the excursion number.

\subsection{LMC X-3}

The long-term X-ray luminosity of the Large Magellanic Cloud black hole candidate LMC X-3 is strongly modulated on timescales of hundreds of days (Cowley et al. 1991; Wilms et al. 2001). The mean 2$10 \mathrm{keV}$ X-ray flux varies by a factor of more than 100 during this long-term cycle (Boyd et al. 2000). While this variability was originally attributed to the precession of a bright, tilted, and warped accretion disk, the discovery of recurrent low/hard states in LMC X-3 argues against this mechanism being responsible for the long-term variation (Wilms et al. 2001). Wilms et al. (2001) report recurrent low/hard states in LMC X-3 based on long-term monitoring with RXTE over several years. This was confirmed by more recent RXTE observations during a low/hard state (Boyd et al. 2000) in which both the timing and spectral characteristics of black hole candidates in the low/hard state was observed. The source is well described by a pure power law with $\Gamma=1.7$ when truly in the low/hard state.
Recently, Brocksopp, Groot \& Wilms (2002) have analyzed 6.5 years of sparsely sampled ground-based optical data on LMC X-3 for long-term modulations. They find no stable long-term period present in the optical data. They find a correlation between the optical and X-ray data with the X-ray lagging the optical by about 10 to 15 days. They explain this as resulting from a variable mass accretion rate that first starts at the outer edge of the disk (and thus apparent in the optical) and moves to the inner edge of the disk (generating $\mathrm{X}$-rays) on the viscous time scale of the disk (10 to 15 days). Weak evidence in their data for a one-day lag between the $\mathrm{V}$ and $\mathrm{B}$ data are consistent with this picture.

Paul, Kitamoto \& Makino (2000) reanalyzed the data from several X-ray missions to study the longterm periods in LMC X-3. The results for this source are similar to those they found for Cyg X-2: there is no stable long-term period present. Further, they find "periods" in subsets of the data for LMC X-3, including, in days, 105, 169, 328, 96, 222, and 178 .

The ASM light curve for LMC X-3 is shown in Figure 4a, where one-day ASM average count rate is plotted against time (JD). If only the first 200 days or so of data are considered, then a modulation with a period close to the Cowley period of 99 (or 198) days appears present. Thereafter, the time evolution of the X-ray flux strongly deviates from strictly periodic. For comparison with Paul, Kitamoto \& Makino (2000), as well as with the Cyg $\mathrm{X}-2$ results presented above, in Figure $4 \mathrm{~b}$ we show an average power spectrum generated by combining the power spectrum from the first half of the ASM data set with that of the second half. The powers are normalized to the Fisher distribution as discussed above. The dashed horizontal line indicates the 5- $\sigma$ detection threshold. The highest peak above this threshold corresponds to a period of 198 days, and is most significant in the second half of the data set. Also significant is a component with a 54-day period. Note that, even though the time series is clearly not strictly periodic with a 198 day period, a simple power spectrum analysis returns a feature at this period with impressive significance.

The mean count rate of the LMC X 3 ASM light curve is $\mu=1.64$ count $\mathrm{s}^{-1}$, with a standard deviation $\sigma=0.92$ count $\mathrm{s}^{-1}$. We use these values to set a threshold for deep minima in LMC X 3 analogous to those in Cyg $\mathrm{X}-2$ as shown by the horizontal line in Figure 5. Times when the X-ray flux stays below $\mu$ - 
$\sigma$ for at least five days have their midpoints marked by vertical lines in Figure 5. Excursions in LMC X-3 are simply the times between adjacent minima. Excursion start times and durations are listed in Table 2.

We refer to the excursions as $P_{i}$, such that $P_{1}$ is the time between the first and second minimum (116 days), $P_{2}$ is the time between the second and third minimum (76 days) and so on. No single excursion $\mathrm{P}_{i}$ has a value near the Cowley estimates of 99 or 198 days. It is interesting to note however that there are two instances when two successive excursion durations add up to a total quite close to the 198 day period (excursions $P_{1}+P_{2}=192$ days, while excursions $P_{6}+P_{7}=204$ days.)

An earlier study of the time evolution of the Xray flux of LMC X-3 based on ASM data up through JD2451230 (Boyd \& Smale, 2000) showed that while there is no simple or multiple periodicity in the system, the excursion lengths were related to each other by simple fractional relations. For example, to within the errors of the period estimates using daily average fluxes, relations such as $P_{2}+P_{3}=P_{4}, P_{6}=P_{1}$, and $P_{7} / P_{8}=4 / 3$ could be found for all excursions in the ASM data available at that time. The subsequent $\mathrm{X}$-ray flux evolution in 1.3 additional years of ASM data for LMC X-3 continue to follow this rule: in fact, every excursion to date can be related to other excursions by rational fractions. The fractional relations for LMC X-3 excursions are listed in Table 3 , where we also note the deviation of the actual fractions of one excursion over another from the rational fraction. Such behavior suggests that there is a natural underlying clock in the system that sets a time scale, and the excursions are simple multiples of this time scale.

The longest excursion, $P_{9}=339$ days is followed by a series of excursions whose lengths are much shorter than earlier excursions (Table 2). The specific fractional relations of excursions $P_{10}$ to $P_{15}$, and $P_{12}$ to $P_{9}$, combined with other fractional relations between the earlier and later excursions suggest that the fundamental clock in the system has a period which is half the value of the shortest excursion to date $\left(P_{10}\right)$. Demanding that integer multiples of this clock period closely reproduce all the LMC X-3 excursions results in a fundamental period $P_{0}=10.594$ days.

Recall the transient periods found by Paul, Kitamoto \& Makino (2000) in subsets of the ASM data on LMC X-3, i.e., in days: $105,169,328,96,222$, and 178. As is the case with our excursions, each of these periods is nearly divisible by $P_{0}=10.594$ days resulting in the following integers: $10,16,31,9,21$, 17.

The X-ray flux variability of LMC X-3 thus joins that of Cyg $\mathrm{X}-2$, being completely characterized as a series of excursions whose lengths are all integer multiples $N_{i}$ of a constant $P_{0}$. While in the case of Cyg X-2 $P_{0}$ is the binary orbital period, there is no known geometric period of 10.594 days in LMC X -3 . Some physical process besides the binary orbit must give rise to the repeating 10.594 day time scale in LMC X-3. From the series of excursions studied to date, it again does not appear that the value of $N_{i}$ can be predicted from knowledge of the sequence of $N_{0}$ through $N_{i-1}$.

\subsection{Cyg $\mathrm{X}-3$}

Much of the variability analysis for Cyg $\mathrm{X}-3$ is concerned with measuring its fast orbital decay, presumed due to a high mass loss rate, and searching for conclusive information about the nature of the companion star and/or the compact object. Fairly frequent radio outbursts also generate many multiwavelength investigations. These outbursts occur on the order of once every 18 months and are accompanied by jets, presumed relativistic, and radio fluxes in excess of 3 Jansky (McCollough et al. 1999). Studies of the correlations between the radio, soft X-ray and hard X-ray flux for Cyg X-3 show strong correlation between radio and soft $\mathrm{X}$-ray, and distinct anti-correlation of the soft and hard X-rays during the low/hard state, suggesting a link between the inflowing matter in the accretion structure and the outflowing jet (Choudhury et al. 2002). No long-term periodicity or time scale as been proposed for Cyg $\mathrm{X}-3$.

The ASM light curve of Cyg $\mathrm{X}-3$ is shown in Figure $6 \mathrm{a}$, where the one-day average rates are plotted against JD. Cyg X-3 shows large amplitude variability in the ASM, reaching intensities as high as 30 count $\mathrm{s}^{-1}$ and as low as 5 count $\mathrm{s}^{-1}$ on timescales of roughly hundreds of days. Shorter term variations are also evident in the ASM light curve. While the longterm X-ray flux from Cyg X 3 in the ASM is clearly not strictly periodic from inspection of Figure $6 \mathrm{a}$, we generated an average power spectrum for comparison with the previously discussed two sources. The power spectrum generated by combining the power spectrum from the first half of the ASM data set with that 
of the second half is shown in Figure $6 \mathrm{~b}$. The powers are normalized to the Fisher distribution as discussed above. The dashed horizontal line indicates the 3$\sigma$ detection threshold. Other than the peak whose period corresponds to the length of the dataset (implying that the input dataset is not stationary), the most significant feature above this threshold corresponds to a central period of $\sim 202$ days. Note that, for a data set short compared to the frequency of interest, the frequency resolution is low. In either half of the data set there are only five cycles of this period, and the uncertainty in the period is $\sim$ one month for a traditional FFT.

Considering the intriguing time spacing of the $\mathrm{X}$ ray minima uncovered in Cyg $\mathrm{X}-2$ and $\mathrm{LMC} \mathrm{X-3}$, we searched for simple relationships between the times of minimum X-ray intensity of a small subset of the light curve of Cyg X-3. We define a minimum in Cyg X3 as a turning point occurring with a flux below 8.0 counts $\mathrm{s}^{-1}$. The minimum centered at JD 2451867 occurs 142 days after the minimum at JD2451725, which itself occurs 213 days after the minimum at JD2451512. The ratio 213:142 is a 3:2 relation with 71 days being the common denominator. With this value as a candidate constant term, we next defined an excursion to have occurred if, between successive minima, the flux of Cyg $\mathrm{X}-3$ rose above 10 counts $\mathrm{s}^{-1}$.

The ASM light curve for Cyg X-3 is plotted again in Figure 7, with the threshold for minimum shown as a horizontal dashed line. The vertical lines are plotted at the midpoint of each minimum. The times of minimum, excursion length and $N_{i}$ for Cyg X-3 are listed in Table 4. There is one instance of $N=1$ (71 days), four instances of $N=2$, two instances of $N=3$, one occurance of $N=4$, and two instances of $N=5$. For this system, the sample size ( 10 excursions) is too small to determine if the distribution of integers is consistent with that found in Cyg X-2. However it is clear that all Cyg X-3 excursions to date can be well described as the product of a small integer multiplied by the constant $P_{0}=71$ days. The $\mathrm{X}$-ray flux variability of Cyg X-3 thus joins that of Cyg X-2 and LMC X-3 as being completely characterized as a series of excursions whose lengths are all integer multiples $N_{i}$ of a constant $P_{0}$. Several VLBA images, spaced a few days apart, and taken after the peak of a $10 \mathrm{Jy}$ flare in Cyg X-3 on 4 February 1997, (Mioduszewski et al. 2001) show a curved, one-sided relativistic jet. If one assumes that the observed curvature arises from precession of the jet, a restricted set of solutions to the geometry and precession period of the disk can be obtained by requiring that the time-separated images be fit simultaneously with the same system geometry and precession period. Mioduszewski et al. (2001) arrive at a period range for the jet precession of $\gtrsim 60$ days. This lower limit to the precession period is consistent with the 71-day timescale we uncovered in the ASM light curve. In Cyg $\mathrm{X}-3$ then, the stable clock may in fact be set by repeating geometry set by the precession of the compact object.

From the series of excursions studied to date, it again does not appear that the value of $N_{i}$ can be predicted from knowledge of the sequence of $N_{0}$ through $N_{i-1}$.

\section{Discussion: Instabilities and Geometry}

The ultimate goal of the study of long-term variability in X-ray binaries is a better understanding of accretion onto compact objects that can explain (and predict) the observed characteristics across a wide variety of sources. Since galactic black hole binaries and neutron star binaries have very similar gravitational potentials, their accretion flows can be expected to have many features in common. Based on early, shorter, and often sparse data sets, the warped, precessing accretion disk model was developed to explain long-term variability in such systems. Strong support for this model could be found in some well-studied systems whose long-term variabilty was nearly strictly periodic, especially SS 433 in the optical, and Her X-1 in the X-ray. Over the past 15 years, several orbiting observatories have collected long, evenly sampled, high $\mathrm{S} / \mathrm{N}$ light curves of numerous $\mathrm{X}$-ray binaries which show long-term variability at time scales longer than the binary orbit. As more cycles of the candidate long-term periods are collected, many sources are found not to show strictly periodic behavior. Here, we have shown that Cyg X-2, LMC X-3 and Cyg X-3 all contain simultaneous periodic and random components in their long-term evolution.

Based on the above analysis, one can postulate a general rule describing the long-term evolution of all three binary systems Cyg X -2 , LMC X -3 , and Cyg $\mathrm{X}-3$. In any particular system, the times between minima of the excursions are related to each other by a constant (which is unique to the system), multiplied by an integer of low order. The distribution of integers is consistent with random: knowledge of the 
previous value(s) of $N$ give no apparent predictive power of the next value of $N$. But can this behavior be reconciled with any of the systems which show well-behaved long-term variability?

To search for such common features, we turned our attention to the long-term evolution of Her $\mathrm{X}-1$. This bright accreting pulsar has a fairly reliable 34.8 day period in its X-ray flux (Scott \& Leahy 1999, Levine \& Jernigan 1982). The system also experiences little understood "anomalous low states" when the longterm period is virtually unobservable and the flux of the system drops dramatically. The best studied example of this began around JD2451250 and was well observed by the RXTE/ASM (Figure 8). The amplitude of the long term period is quenched suddenly over one cycle, and restarts just as abruptly.

The turn-on times of the 35-day cycle have been observed to be tied to the orbital phase of the binary in an unusual way, occuring at either $\phi=0.2$ or $\phi=0.7$. In nearly every case, the cycle length (defined as the time between adjacent turn-on times) is either $20,20.5$ or 21 binary orbits (see Scott \& Leahy 1999, Table 1). Scott \& Leahy (1999) find that these half-orbital phase jumps appear to be randomly distributed.

The Her $\mathrm{X}-1$ cycle lengths are exactly analogous to the excursion times discussed in this paper. In fact, the excursions in Her $\mathrm{X}-1$ can be considered to be described as the product of a random integer $N_{i}$ (which in this case clusters about the values $N=39,40,41$, 42 ) and a constant $P_{0}=P_{\text {binary }} / 2$. In this case, while the values of $N$ appear to be randomly distributed, they favor a small number of values. Thus the longterm variability in Her $\mathrm{X}-1$ is generally consistent with the rule we found in Cyg X-2, LMC X-3 and Cyg X-3.

The long-term light curves of all four systems appear to have gross features in common: the times between deep minima can be described as the product of an underlying constant and a random integer. The constant varies from system to system, but is tied to the binary orbit in two (Cyg $\mathrm{X}-2$ and Her $\mathrm{X}-1$ ). With this general behavior in hand, it is now possible to seek a model for accretion in binary systems. The model must contain this common feature across a broad range of system parameters.

Other systems in nature share this property of simultaneous random and periodic components, such as the times between hurricanes classified as Cate- gory 4 or stronger to strike the North Carolina coast (Landsea 1993, Jarrell et al. 2001). Intense hurricanes originating in the Atlantic basin display a significantly more peaked distribution than weaker cyclones, with $95 \%$ of intense hurricane activity occurring between August and October. In this case, the statistics of the distribution of times between intense events are controlled by the combination of Earth's orbital period (constant) and Earth's turbulent atmosphere (random). An outside observer armed only with one observational variable tracking Atlantic hurricane intensity would record a time series evolution similar to those of the X-ray binaries in the current study: a periodic component does exist, but the behavior does not repeat each period. Instead the observer must wait some random number $N$ of periods $P$ before the behavior repeats. This implies that at each $P$ a condition recurs which is necessary, but not sufficient, to cause the observed behavior. The weather patterns do not repeat exactly from year to year because the atmosphere of Earth is complex, and turbulent, giving rise to the random component in the distribution function of intense hurricanes.

All X-ray binary systems share two general features, i.e., a regular binary period and a turbulent accretion process, generally through a disk. Can the long-term variations in X-ray binaries be considered disk weather?

\subsection{Topological Analysis of the Time Series}

There are several plausible physical processes that might give rise to the random component in X-ray binary systems, such as disk magnetic fields, the dynamics of the companion star atmosphere, and/or the dynamics of coherent structures which may form in the accretion disk. At the same time there are many ways in which one might tie these processes to a periodicity in the system when developing model equations, such as the location on the disk where the interaction is most important, as well as the exact mathematical form of the coupling. Specific physical mechanisms which may be important in accretion disk evolution include the hydrogen ionization instability, the temperature at which neutral hydrogen is ionized (Lasota 2001), advective cooling, and the Balbus- Hawley magnetic instability (Balbus \& Hawley 1991).

In order to extract all available dynamical information from the one-dimensional time series of soft $\mathrm{X}$-ray flux versus time, we employed a method which has been fruitful in the analysis of time series from 
driven, dissipative systems in a variety of physical processes. The so-called topological analysis method (see Gilmore 1998 and numerous references therein) relies on embedding the one-dimensional time series in phase space, and extracting the topological properties of motion of unstable periodic orbits on the manifold upon which the dynamics is occurring. Embedded in the phase space manifold on which the system evolves are the fingerprints of the underlying unstable periodic orbits. These orbits twist and turn about one another in a way that communicates the topology of the manifold.

Classes of dynamical systems, differing in the types of coupling between nonlinear equations, give rise to distinct topological properties in the phase space. Inverting this statement, if topological properties of the relevant phase space can be extracted from a onedimensional time series in this way, their existence prunes the space of possible physical models - the topological properties will be consistent with some forms of coupled equations, and inconsistent with others.

We thus embedded the ASM light curves into a realistic two-dimensional phase space. Assuming the soft X-ray rate $X_{i}$ carries information about an important dynamical variable in the system (generally assumed to be the instantaneous mass accretion rate), we can represent the time rate of change of this variable by computing the instantaneous slope of $X_{i}$ at each discrete time, i. e., $Y=\dot{X}$. In all cases, since we are interested in the low frequency component of the ASM light curves, we filtered the input data to remove frequencies $>0.2$ day $^{-1}$. Filtering was done by Fourier transforming the original data, removing the highest frequencies, and then back-transforming to the time domain. The filtered light curve of Cyg $\mathrm{X}-3$ is shown in Figure 9. The vertical lines show segments that were extracted as surrogates for the unstable periodic orbits of 1,2 and 3 times $P_{0}=71$ days. Note in the time series that the number of maxima between global minima is equal to the order of the orbit. These orbits are shown embedded in $X, \dot{X}$ phase space in Figure 10. In this phase space, two regions can be identified about which the trajectories evolve.

The phase space embeddings for several orbits are shown for LMC X 3 in Figure 11 and Cyg X-2 in Figure 12. In each case there are two regions of the phase space about which the orbits evolve: one which corresponds to a minimum in the light curve with a relatively high $\mathrm{X}$-ray rate, and the other with a lower
$\mathrm{X}$-ray rate (the global minimum in each system).

This phase space evolution is similar to many nonlinear dynamical system, and markedly disimilar to random or periodic behavior. For a random time series, even short segments embedded in phase space would tend to fill the space more uniformly, while an embedded periodic signal would trace out a single closed curve. On the other hand, many canonical nonlinear dynamical systems exhibit two phase space regions about which the flow is organized, including the driven Duffing oscillator, and the Lorenz system.

The equations describing the driven Duffing system are

$$
\begin{aligned}
& \dot{x}=v \\
& \dot{v}=x-x^{3}-b v+A \sin \phi \\
& \dot{\phi}=\omega
\end{aligned}
$$

The system has a linear repulsive term, cubic nonlinear restoring force, a damping term proportional to the velocity, and a periodic driving term. This system has a double-well potential: the origin is unstable, and given an arbitrary perturbation and no driving, the system either settles into one potential minimum or the other. The one-dimensional time series of the driven system $x(t)$, obtained by integrating the equations of motion for a fixed set of parameters $A$ and $\omega$, shows minima occurring about two values, one with a higher amplitude and the other with lower amplitude value. In Figure 13 a phase space projection of the driven Duffing system is shown, which has two regions of phase space about which the trajectory evolves, similar to what was seen in the X-ray flux light curves. Each location corresponds to one of the potential wells-in effect there is a competition between these two solutions for control of the time evolution of the system. In analogy with the Duffing system, two competing states in accretion disks could plausibly arise due to: a geometric symmetry about the flat disk, in which the disk is likely to settle into either a warp-up or warp-down configuration; the competition between high/soft and low/hard accretion states; competing warping modes (Ogilvie \& Dubus, 2001); or magnetic field reversals within the disk.

\subsection{Disk Magnetic Fields}

Spectral studies of X-ray systems show broad similarities between accreting neutron star and black hole candidate systems (see the review by Done 2002 and references therein). In each case, a full description of 
the $\mathrm{X}$-ray spectrum requires a hard-tail component, which can be explained by invoking the same mechanism that is thought responsible for the disk viscosity, namely the Balbus-Hawley MHD dynamo (Balbus \& Hawley 1991). In this scenario, when a disk possesses ionized Hydrogen the MHD dynamo operates and localized regions of magnetic reconnection at the surface of the disk causes sufficient electron heating to generate the hard X-ray tail.

A similar physical model, the Rikitake disk dynamo (Rikitake 1958, Seki \& Ito 1999) has been favored to explain the statistical distribution of Earth's magnetic field reversals. Under certain parameter ranges, this system of equations has been shown to undergo "Lorenz-type" nonlinear evolution, with projections of the flow onto two-dimensional phase space planes showing two regions organizing the flow (McMillen 1999).

The equations of the Rikitake disk dynamo system are well known. The existence of generally similar behavior in the organization of trajectories in the space space for the X-ray binaries, and for the numerically generated Rikitake model means that it is possible to perform a full topological analysis on the observational data. This involves extracting the topological invariants from the observational data, organizing those for each observed system into a template (Mindlin et al. 1991, Gilmore 1998) and then determining if the individual templates are equivalent from system to system. If so, the same procedure can be followed using numerically generated data from a disk dynamo model, or any other realistic physical model, and its template compared to the data. Finding an equivalent template in the model data would validate the model, whereas a template with a different underlying topology than the observational template would mean the model does not accurately explain the observed behavior. This statement is powerful, as it implies that even astronomical data that is not periodic can be used to validate or refute intricate dynamical models for accretion disks.

\section{Conclusions}

We have found that the long-term $\mathrm{X}$-ray variability of Cyg X -2, LMC X-3 and Cyg X-3 can be described only with the simultaneous existence of periodic and random components. In each case, the times between $\mathrm{X}$-ray minima are related to each other by a constant, unique to the system, multiplied by a low-order inte- ger, the values of which are apparently randomly distributed. Two of these systems were historically presumed long-term periodic (Cyg X-2, and LMC X-3) and the third (Cyg X-3) has no reported X-ray periodicity on time scales of hundreds of days. In each of these cases, the long-term evolution is best described by the same mathematical rule-the simultaneous existence of a stable clock in the system and a simple random component. If the overall $\mathrm{X}$-ray intensity is coupled to differing states of the accretion disk (optically thick versus thin for example) then the existence of this mathematical rule implies a fixed probability for disk transition at every clock iteration. At each clock iteration, a necessary, but not sufficient, condition repeats; there is a stable clock but a randomly set alarm. In Cyg X-2, the underlying constant is simply the 9.844-day binary period. In Cyg X-3, the 71 day constant is much longer than the 4.8 hour binary orbit, but similar to the inferred precession period of the radio jet. There is no known geometric period in LMC $\mathrm{X}-3$ at the observed 10.594 day timescale. The same general description applies to the well-known orbital phase dependence of the turn-on times in Her X-1, which can be described as the product of a random distribution of integers multiplied by half the binary orbital period.

Finding this common, unifying behavior in the time evolution of three generally different X-ray binary systems suggests it is tied to a fundamental feature of the accretion process onto compact objects in binary systems, and is not related to the nature of the compact object itself (neutron star or black hole).

Previous explanations of long-term variability, which invoke either system geometry (a warped, precessing disk) or disk instabilities as causes of the X-ray variability may need to be combined in order to build a complete theoretical picture of accretion onto compact objects via accretion disks. The similarity of the phase space evolution of the X-ray binary trajectories with several well known dissipative dynamical systems suggests that exploring the topological properties of the data, and comparing these to the models, can focus the efforts of developing a complete model of accretion disk dynamics and its connection to longterm variability in $\mathrm{X}$-ray binaries in general.

This research has made use of data obtained through the High Energy Astrophysics Science Archive Research Center Online Service, provided by NASA's Goddard Space Flight Center. ASM results were pro- 
vided by the ASM/RXTE teams at MIT and at the RXTE SOF and GOF at NASA's GSFC. We thank Drs. J. F. Heagy, and J. C. Cannizzo for several useful discussions and suggestions.

\section{REFERENCES}

Abell, G. O. \& Margon, B. 1979, Nature, 279, 701

Balbus, S. A. \& Hawley, J. F. 1991, ApJ, 376, 223

Boyd, P. T., Smale, A. P., Homan, J., Jonker, P. G., van der Klis, M. \& Kuulkers, E., 2000, ApJ, 542, L127

Boyd, P. T. \& Smale, A. P. 2000, Rossi2000: Astrophysics with the Rossi X-ray Timing Explorer, $p$ E20

Bradt, H. V., Rothschild, R. E., \& Swank, J. H. 1993, A\&AS, 97, 355

Brocksopp, C., Groot, P. J. \& Wilms, J. 2001, MNRAS, 328, 139

Casares, J., Charles, P. A. \& Kuulkers, E. 1998, ApJ, 493, L39

Choudhury, M., Rao, A. R., Vadawale, S. V., IshwaraChandra, C. H. \& Jain, A. K. 2002, A\&A, 383, L35

Cowley, A.P., Crampton, D., \& Hutchings, J.B., 1979, ApJ, 231, 537

Cowley, A.P., Crampton, D., \& Hutchings, J.B., 1983, ApJ, 272, 118

Cowley, A. P., Schmidke, P. C., Ebisawa, K., Makino, F., Remillard, R. A., Crampton, D., Hutchings, J.

B., Kitamoto, S. \& Treves, A., 1991, ApJ, 381, 526

Done, C. 2002, preprint (astro-ph/0203246 v1)

Ebisawa, K., Makino, F., Mitsuda, K., Belloni, T., Cowley, A. P., Schmidke, P. C. \& Treves, A., 1993, ApJ, 403, 684

Fabian, A. C. \& Rees, M. J. 1979, MNRAS, 187, 13p

Fisher, R. A., 1929, Proc. R. Soc., A., 125, 54

Gilmore, R. 1998, Reviews of Modern Physics, 70, 1455

Gruber, D.E. \& Rothschild, R. E. 1984, ApJ, 283, 546

Hanson, M. M., Still, M. D \& Fender, R. P. 2000, ApJ, 541, 308

Jarrell, J. D., Mayfield, M., Rappaport, E. N. \& Landsea, C. W. 2001, NOAA Technical Memorandum NWS-TPC-1

Kahn, S. M. \& Grindlay, J. E. 1984, ApJ, 281, 826
Kong, A. K. H., Charles, P. A. \& Kuulkers, E. 1998, NewA, 3, 301

Landsea, C. W. 1993, Monthly Weather Review, 121, 1703

Lang, F. L. et al. 1981, ApJ, 246, L21

Lasota, J.-P. 2001, New Astronomy Reviews, 45, 449

Levine, A. M., \& Jernigan, J. G. 1982, ApJ, 262, 294L

Levine, A. M., Bradt, H., Cui, W., Jernigan, J. G., Morgan, E. H., Remillard, R., Shirey, R. E., \& Smith, D. A. 1996, ApJ, 469, L33

Mazeh, T., van Paradijs, J., van den Heuvel, E. P. J., Savonije, G. H., 1986, A\&A, 157, 113

McCollough, M. L., Robinson, C. R., Zhang, S. N., et al. 1999, ApJ, 517, 951

McMillen, T. 1999, The Nonlinear Journal, 1, 1

Mindlin, G. B., Solari, H. G., Natiello, M. A., Gilmore, R. \& Hou, X-. J. 1991, J. Nonlinear Sci. 1, 147

Mioduszewski, A. J., Rupen, M. P., Hjellming, R. M., Pooley, G. G. \& Waltman, E. B. 2001, ApJ, 553, 766

Mitra, A. 1998, ApJ 499, 385

Ogilvie, G. I. \& Dubus, G. 2001, MNRAS 320, 485

Orosz, J. A. \& Kuulkers, E. 1999, MNRAS, 305, 132

Paczynski, B., 1983, ApJL, 273, 81

Paul, B., Kitamoto, S. \& Makino, F. 2000, ApJ, 528, 410

Priedhorsky, W. C. \& Holt, S. S. 1987, Space Sci. Rev. E, 45, 291

Rikitake, T. 1958, Proc. Camb. Phil. Soc., 54, 89

Seki, M. \& Ito, K. 1999, Earth Planets Space, 51, 395

Schmutz, W., Geballe, T. R. \& Schild, H. 1996, A\&A, 311, L25

Scott, D. M. \& Leahy, D. A. 1999, ApJ, 510, 974

Shimshoni, M., 1971, Geophys. J. R. astr. Soc., 23, 373

Smale, A. P. 1998, ApJ, 498, L141

Smale, A. P. \& Lochner, J. C. 1992, ApJ, 395, 582 van der Klis, M. \& Kuulkers, E., 2000, ApJ, 542, L127

Vrtilek, S. D., Swank, J. H., Kelley, R. L. \& Kahn, S. M. 1988, Ap.J, 329, 276 
Waltman, E. V. Ghigo, F. D., Johnson, K. J., Foster, R. S., Fiedler, R. L. \& Spencer, J. H. 1995, AJ, 110,290

Warren, P. R. \& Penfold, J. E. 1975, MNRAS, 172, 41P

Wijnands, R. A. D., Kuulkers, E. \& Smale, A. P. 1996, ApJ 473, L45

Wilms, J., Nowak, M. A., Pottschmidt, K., Heindl, W. A., Dove, J. B., \& Begelman, M. C. 2001, MNRAS, 320,327

Wojdowski, P., Clark, G. W., Levine, A. M., Woo, J. W. \& Zhang, S. N. 1998, ApJ, 502, 253

This 2-column preprint was prepared with the AAS IATEX macros $v 4.0$.
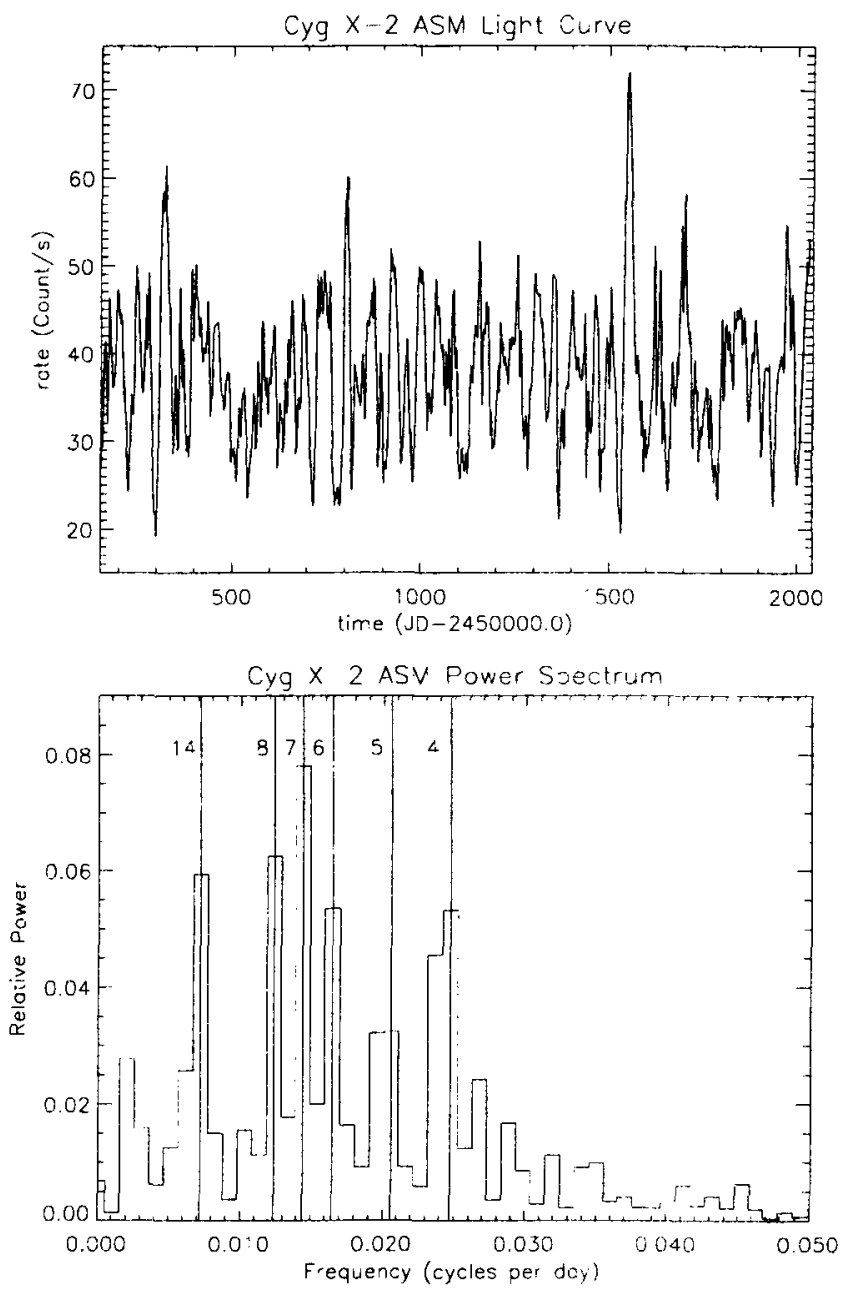

Fig. 1.- A. All Sky Monitor Long Term Light Curve for Cyg X-2 ( 1 day bins). The 78-day "period" in the earliest data is followed by excursions whose amplitudes and durations vary with time. B. Power Spectrum of the ASM data created by averaging the power spectra of the first and second half of the data set. The highest peaks in the power spectrum are marked with vertical lines, which are labeled as integer multipes of the 9.844 day binary period. 
TABLE 1

Cyg X-2 Period Multiples and Corresponding Frequencies

\begin{tabular}{lll}
\hline $\mathrm{N}$ & $\mathrm{N}^{*} \mathrm{P}($ days $)$ & $(N * P)^{-1}(\mathrm{~Hz})$ \\
\hline 4 & 39.38 & 0.025 \\
5 & 49.22 & 0.020 \\
6 & 59.07 & 0.017 \\
7 & 68.91 & 0.015 \\
8 & 78.76 & 0.013 \\
14 & 137.82 & 0.007 \\
\hline
\end{tabular}

Table 2

LMC X-3 Excursions and Multiples of $P_{0}=10.594$ DAYS

\begin{tabular}{lllll}
\hline Excursion & Start Time (JD-2450000.0) & Duration (days) & $\mathrm{N}$ & $\mathrm{N}^{*} \mathrm{P}_{0}$ (days) \\
\hline P1 & 145 & 116 & 11 & 116.5 \\
P2 & 261 & 76 & 7 & 74.1 \\
P3 & 337 & 136 & 13 & 137.7 \\
P4 & 473 & 212 & 20 & 211.9 \\
P5 & 685 & 292 & 28 & 296.6 \\
P6 & 977 & 118 & 11 & 116.5 \\
P7 & 1095 & 86 & 8 & 84.8 \\
P8 & 1181. & 64 & 6 & 63.6 \\
P9 & 1245 & 339 & 32 & 339.0 \\
P10 & 1584 & 21 & 2 & 21.2 \\
P11 & 1605 & 54 & 5 & 53.0 \\
P12 & 1659 & 42 & 4 & 42.4 \\
P13 & 1701 & 43 & 4 & 42.4 \\
P14 & 1744 & 32 & 3 & 31.8 \\
P15 & 1776 & 31 & 3 & 31.8 \\
P16 & 1807 & - & - & - \\
\hline
\end{tabular}


TABLE 3

LMC X-3 Example Rational Fractions Relations

\begin{tabular}{ll}
\hline \multicolumn{1}{c}{ Relation } & Deviation from Rational \\
\hline$P 2+P 3=P 4$ & 0.0 \\
$P 3 / P 1=13 / 11$ & 0.008 \\
$P 4 / P 8=10 / 3$ & 0.006 \\
$P 6=P 1$ & 0.017 \\
$P 7 / P 8=4 / 3$ & 0.008 \\
$P 9 / P 7=4$ & 0.015 \\
$P 8 / P 10=3$ & 0.016 \\
$P 11+P 14=P 7$ & 0.0 \\
$P 12 / P 10=2$ & 0.0 \\
$P 7 / P 13=2$ & 0.0 \\
$P 14 / P 10=3 / 2$ & 0.016 \\
$P 15 / P 10=3 / 2$ & 0.016 \\
$P 9 / P 12=8$ & 0.009 \\
\hline
\end{tabular}

TABLE 4

Cyg X -3 EXCURSions and MUltiples of 71 DAYS

\begin{tabular}{lll}
\hline \hline min time (JD-2450000.0 & excursion length (days) & N \\
\hline 163 & 71 & 1 \\
234 & 142 & 2 \\
376 & 284 & 4 \\
660 & 142 & 2 \\
802 & 142 & 2 \\
944 & 213 & 3 \\
1157 & 355 & 5 \\
1512 & 213 & 3 \\
1725 & 142 & 2 \\
1867 & 355 & 5 \\
2222 & - & - \\
\hline
\end{tabular}



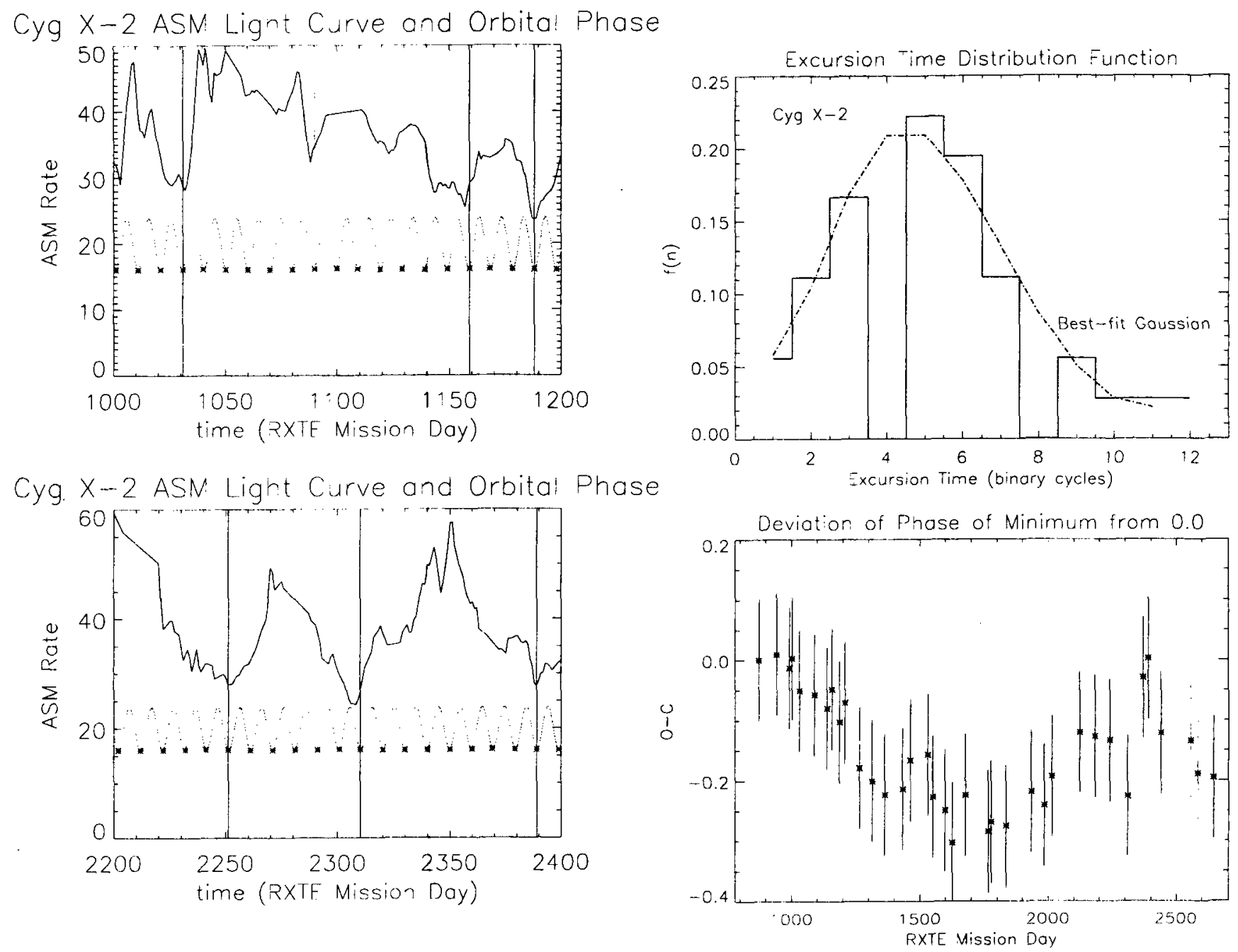

Fig. 2.-- Two short segments of the Cyg X-2 ASM time series demonstrating the definition of "deep" minima (those in which the X-ray flux drops lower than 30 ASM counts $\mathrm{s}^{-1}$ ) and their relation to the binary orbit, the phase of which is represented as the sine wave appearing below the ASM light curve. Each segment shows how the deep minima generally align with binary phase. The number of binary orbits between deep minima appears to be randomly distributed. In general, the entire time series of Cyg $\mathrm{X} 2$ can be characterized as a series of integer multiples of the binary period $\mathrm{N}_{i}$.

Fig. 3.- Statistical properties of the set of integers $\mathrm{N}_{i}$ describing the time between deep minima. The normalized histogram (a) of $N_{i}$, shown with the bestfit Gaussian distribution. The total number of excursions is 36 . The histogram is also well described by a Poisson distribution. The deviations of the binary phase at deep minimum versus time in RXTE mission day (b) implies variations in binary phase of deep minimum are not random, but correlated. 

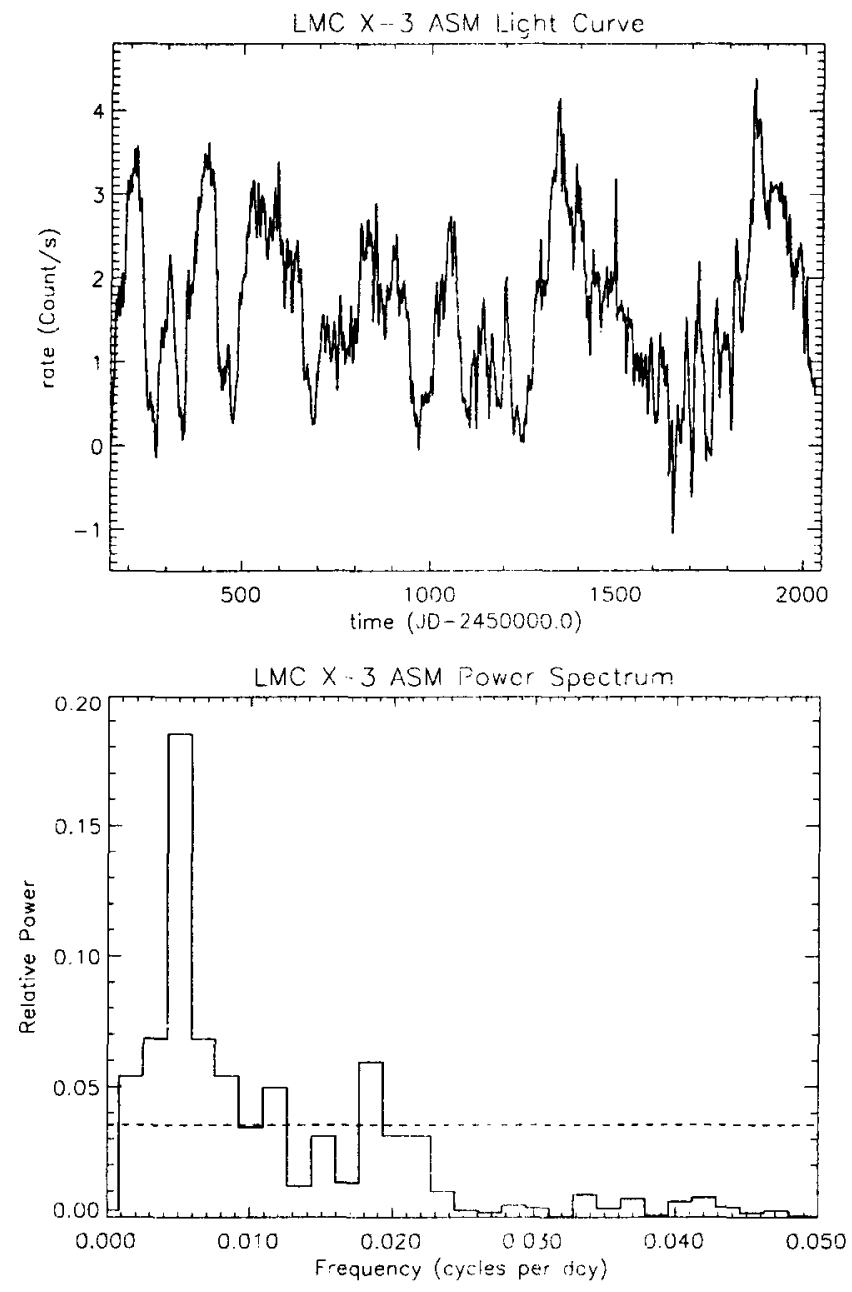

Fig. 4.-- A. LMC X-3 long-term ASM light curve, one day bins. The 99 (or 198) day periodicity detected by Cowley et al. (1991) is apparent in the first 200 days of ASM data. Thereafter, the time evolution is far from simply periodic. The average power spectrum of the first and second halves of the ASM data is shown in $\mathrm{B}$. The dashed horizontal line indicates the 5- $\sigma$ threshold. The most significant frequency corresponds to a period of 198 days, and is most significant in the second half of the data set. The highest significant frequency, more prominent in the first half of the data, has a corresponding period of 54 days.
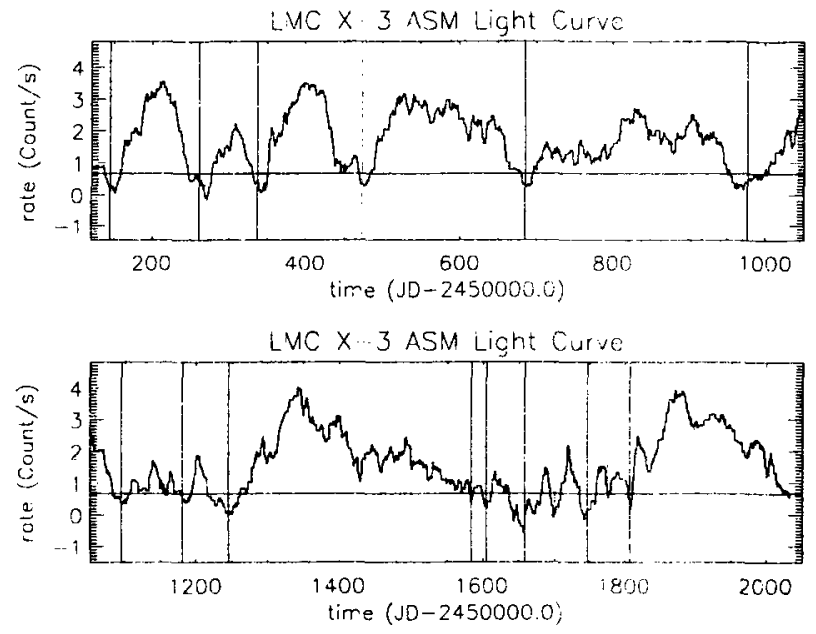

Fig. 5.-- Excursions in the long-term light curve of LMC X-3. The horizontal line at 0.67 count $\mathrm{s}^{-1}$ is the mean minus the standard deviation. Times when the light curve stays below this level for at least 5 days have their midpoints marked with vertical lines. 

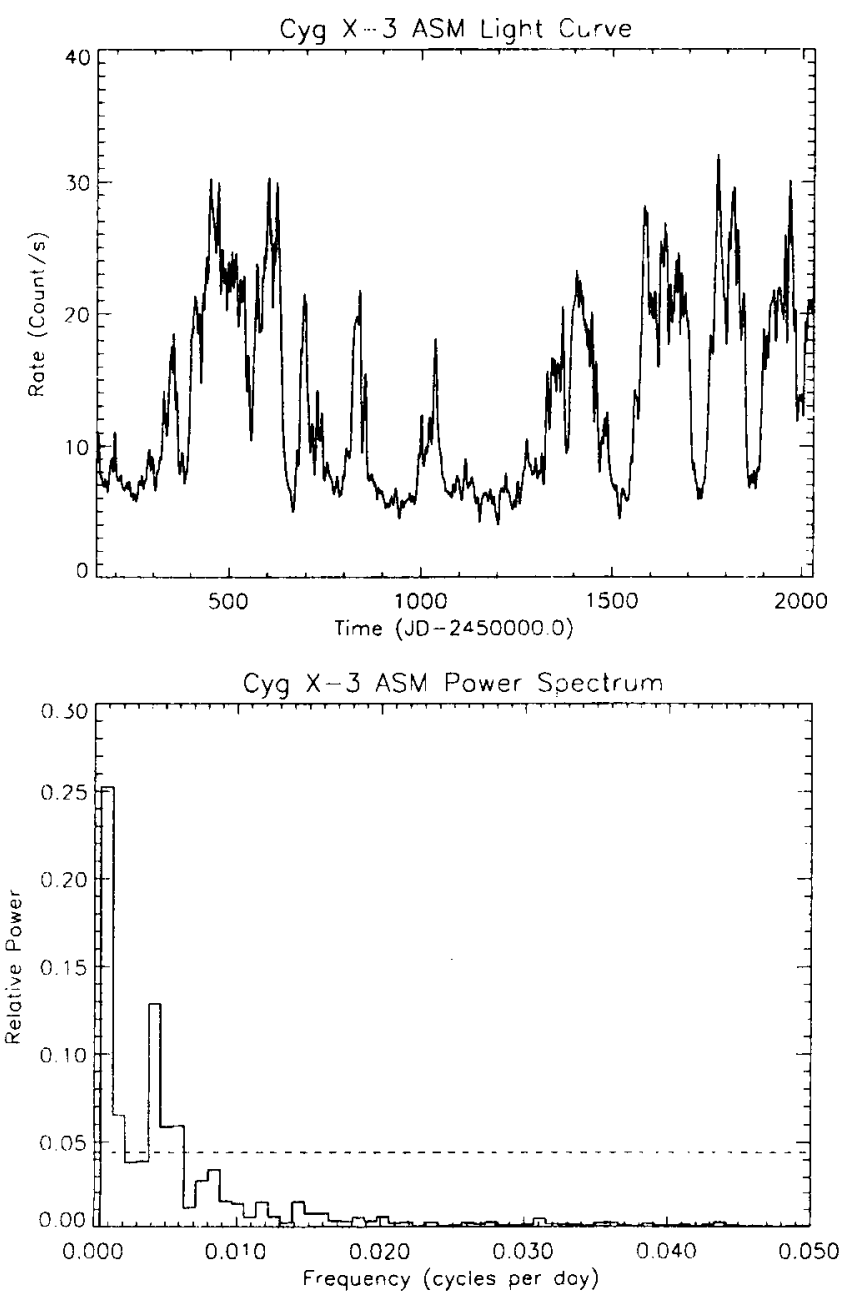

Fig. 6.-- A. The long-term ASM light curve of Cyg $\mathrm{X}-3$. The system repeatedly, though not periodically, becomes as bright as 30 count $\mathrm{s}^{-1}$, and as dim as 6 count $\mathrm{s}^{-1}$. B. The average power spectrum of the first and second halves of the ASM data. The dashed horizontal line indicates the 3- $\sigma$ threshold. The most significant feature corresponds to a period $\sim 202$ days.
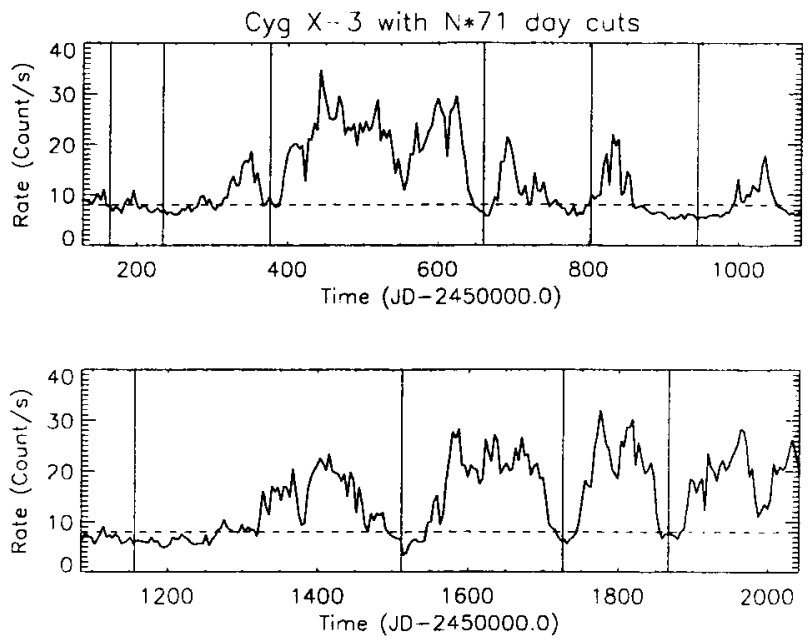

Fig. 7.- Excursions in $\mathrm{Cyg} \mathrm{X}-3$ are defined as the times between adjacent turning points below 8.0 counts $\mathrm{s}^{-1}$, which also contain times when the flux rises above 10.0 counts $\mathrm{s}^{-1}$. These excursions can be quantized in units of $n^{*} 71$ days.
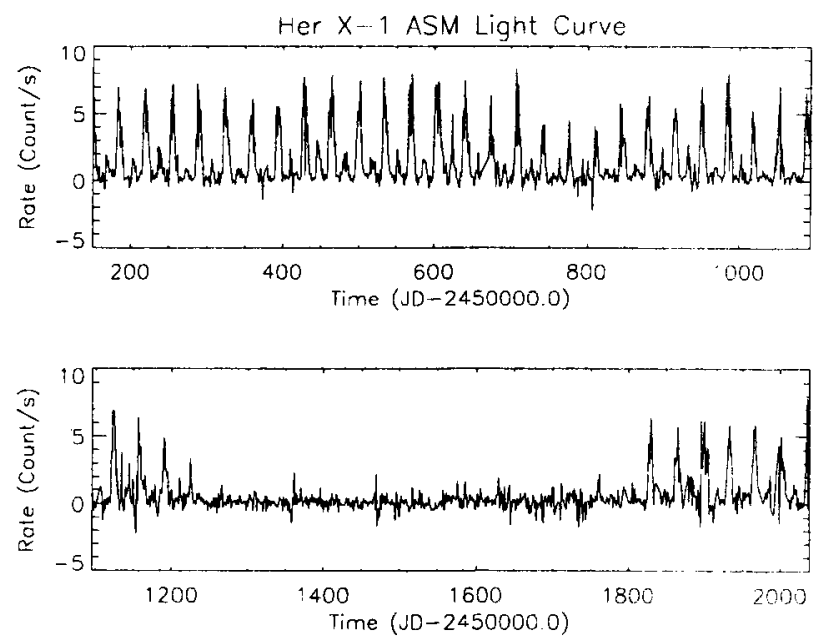

Fig. 8.- ASM light curve of Her $\mathrm{X}-1$, daily averages versus JD. The well-known $\sim 35$-day long-term period is clear in the beginning and end of the light curve. A long, anomalous low state occurs in the middle, where the X-ray flux and amplitude of the long-term period drop dramatically for over one year. 

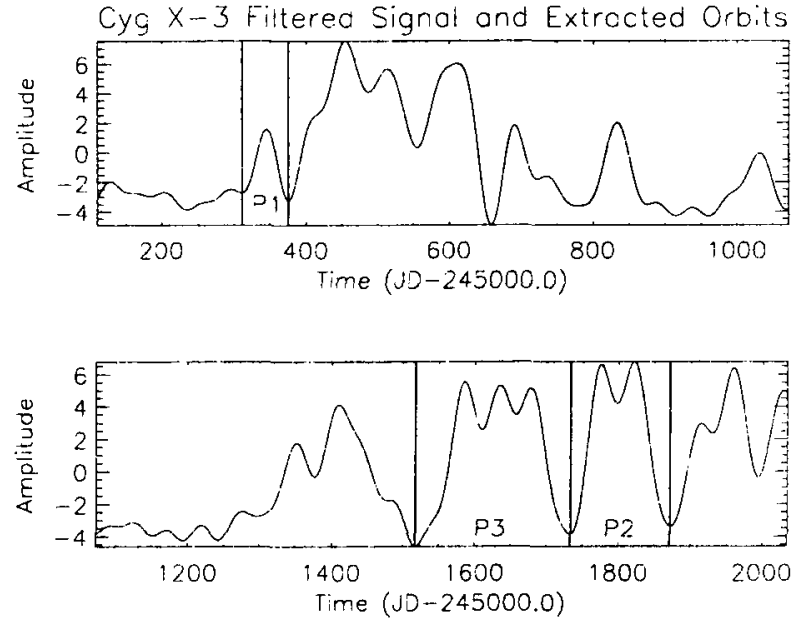

Fig. 9.- ASM light curve of Cyg X-3 with highest frequencies removed. Vertical lines denote segments which were extracted to study the phase space behavior of segments with length 1,2 and 3 times 71 days.
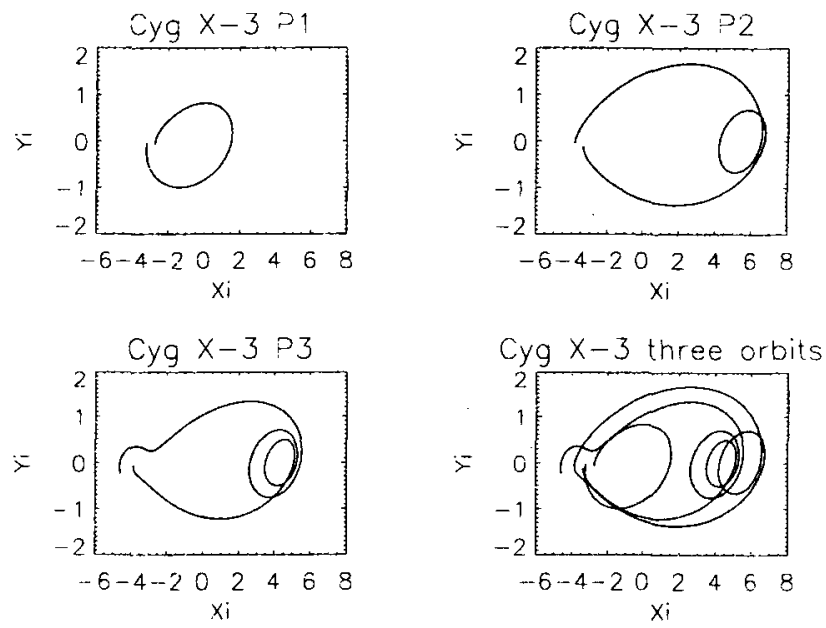

Fig. 10.- Phase space plots of the three extracted segments of data from $\mathrm{Cyg} \mathrm{X}-3$ from Figure 9. In each case, $X_{i}$ is the amplitude of the filtered signal, and $Y_{i}$ is the instantaneous derivative of the signal at each time step, $i$. Top left: the 71 day, "period 1" orbit. Top right: The 140 day, period 2 orbit. Bottom left, the 210 day period 3 orbit. Bottom right: All three orbits superposed show two separate regions of rotation in phase space. This phase space behavior is similar to well-studied dynamical systems contain- ing a bistability in the potential (c.f. the driven Duffing system in Figure 13). 

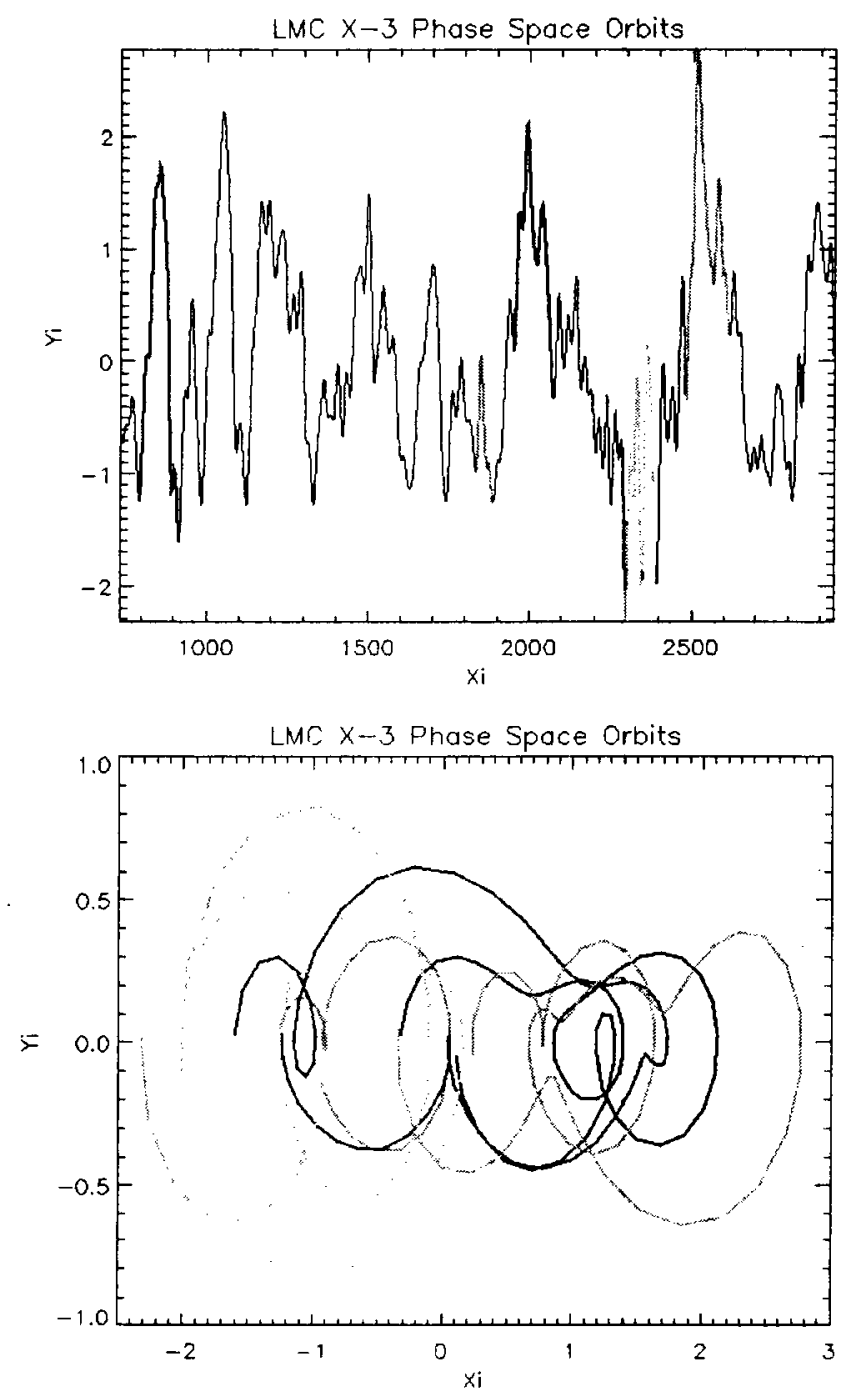

Fig. 11.-- Several segments of the long-term light curve of LMC X-3, shown in A) are plotted in twodimensional phase space, B. Again, trajectories can be seen to rotate about two points in the phase space, distinguished by the count rate at local minimum in the time series.
Cya $x \ldots 2$ tepologicol Aralysis
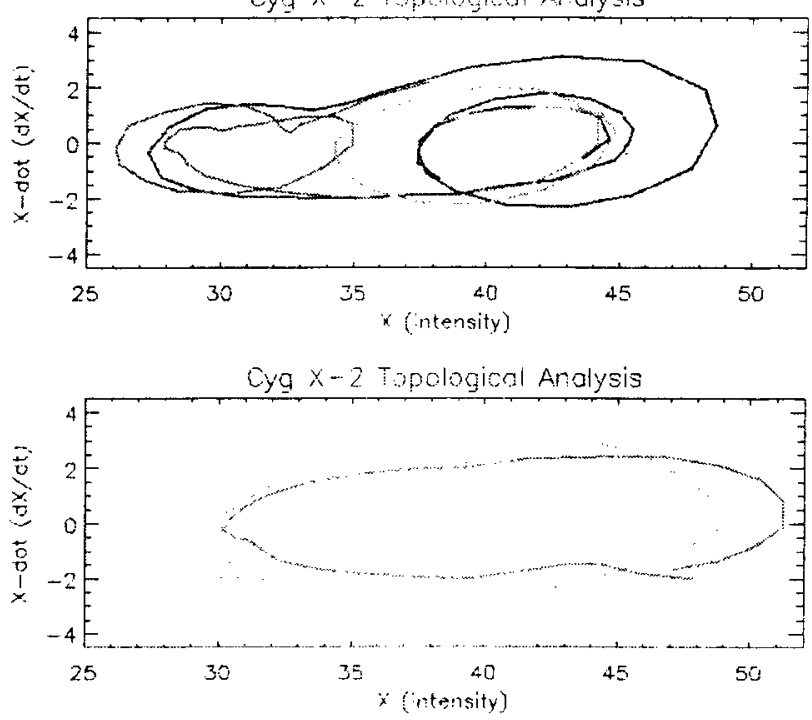

Fig. 12.- Several segments of the long-term light curve of Cyg X-2 are plotted in this phase space. Two fixed points can be seen, around which rotation occurs. That on the right, at higher count rate, corresponds to segments of the time series where a maximum is followed by a minimum with a relatively high intensity at the turning point.

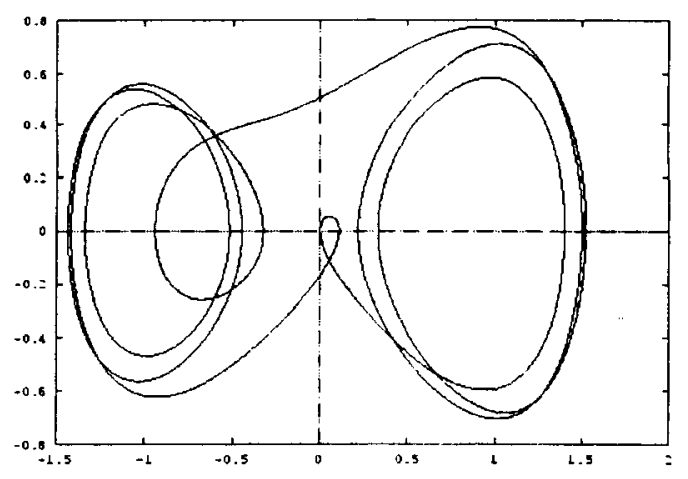

Fig. 13.- A projection of the driven Duffing system in the $(x, \dot{x})$ plane, with $\omega=0.12861$. This asymmetric oscillation shows two regions about which the trajectory evolves. 